\title{
Patient-reported quality of life with interstitial partial breast brachytherapy and external beam whole breast radiotherapy: a comparison using propensity-score matching
}

\author{
Prof. Tabassum Wadasadawala, MD!, Priyamvada Maitre, MD², Shwetabh Sinha, MD², Vani Parmar, MS³, \\ Rima Pathak, MD², Mithila Gaikar, MSc' Shalini Verma, MD², Rajiv Sarin, FRCR ${ }^{2}$ \\ 'Department of Radiation Oncology, Advanced Centre for Treatment Research and Education in Cancer (ACTREC), Tata Memorial Centre, \\ Homi Bhabha National Institute, Navi Mumbai, India, ${ }^{2}$ Department of Radiation Oncology, Tata Memorial Hospital, Homi Bhabha National \\ Institute, Mumbai, ${ }^{3}$ Department of Surgical Oncology, Tata Memorial Hospital, Homi Bhabha National Institute Mumbai, India, ${ }^{4}$ Department \\ of Clinical Research, Tata Memorial Hospital, Homi Bhabha National Institute Mumbai, India
}

\begin{abstract}
Purpose: The aim of this study was to compare patient-reported quality of life (QOL) scores after accelerated partial breast irradiation (APBI) using interstitial brachytherapy vs. external beam whole breast radiotherapy (WBRT) for breast cancer.

Material and methods: Women with breast cancer treated with WBRT or APBI after breast conservation surgery were enrolled in this prospective study. Single cross-sectional QOL assessment was performed using EORTC QLQ-C30 and BR-23 questionnaires. Patients treated with APBI were propensity-score matched to similar cohort of patients treated with WBRT. QOL scores were analyzed for the entire unmatched cohort and compared between the two matched cohorts using Student's two-tailed $t$-test. $P$-value of $<0.05$ was considered statistically significant, and a 10 -point difference between mean scores was considered clinically meaningful.

Results: A total of 64 APBI patients were matched with 99 WBRT patients out of the entire study cohort of 320 cases. QOL scores for functional scales of QLQ-C30 were similar between the two groups for both matched and unmatched cohorts, while symptom scores of QLQ-C30 did not show any clinically significant difference. Functional scales of BR-23 did not show any clinical or statistically significant difference. Among symptom scales of BR-23, scores were similar for APBI and WBRT groups except for a worse score of "upset by hair loss" sub-scale in the brachytherapy group of the matched cohort (51.9 vs. 22.7, $p=0.006$ )

Conclusions: Patients undergoing APBI reported similar QOL compared to WBRT when matched for various factors.

J Contemp Brachytherapy 2021; 13, 4: 387-394 DOI: https://doi.org/10.5114/jcb.2021.107690
\end{abstract}

Key words: APBI, brachytherapy, propensity-score matching, quality of life, breast radiotherapy.

\section{Purpose}

For early breast cancer patients undergoing breast conservation therapy, accelerated partial breast irradiation (APBI) offers the advantage of shorter overall treatment time for appropriately selected patients [1,2]. It is an acceptable alternative to external whole breast radiotherapy (WBRT), with reduced toxicity and improved cosmetic outcomes [3, 4]. Out of various techniques available to deliver APBI, multi-channel nylon catheter-based interstitial brachytherapy is the most established technique [5]. Many studies published physician-reported and patient-reported cosmetic outcomes with APBI in comparison with WBRT $[6,7]$. Comparison of patients' quality of life (QOL) is an integral part of outcomes reporting while evaluating different treatment modalities. Various Western retrospective and prospective randomized studies have shown similar cosmesis and QOL outcomes with APBI and WBRT [8-10]. In the absence of randomized data from Indian population, matching APBI and WBRT patients' cohorts for important factors could improve the quality of comparison of QOL scores. These factors consist of treatment aspects, such as type of primary breast surgery, type of endocrine therapy, receipt of adjuvant chemotherapy, tumor factors, like tumor and nodal stage, and patient factors, including age and menopausal status $[11,12]$. Therefore, this study employed a propensity- 
score matched pair analysis for comparing short-term patient-reported QOL scores after APBI and WBRT in a prospective manner.

\section{Material and methods}

This was a prospective observational cross-sectional study, approved by the institutional review board.

\section{Patients' selection}

Women with breast cancer, who had undergone breast-conserving surgery followed by adjuvant radiotherapy, and attending every 6 months follow-up visits at our institute were screened for this study. These patients who were within a follow-up period of 18 to 36 months post-radiotherapy were enrolled in the present study, after obtaining a written informed consent. Patients with mastectomy, bilateral breast cancer, or reconstructive procedures were excluded from the research. Breast-conserving surgery comprised of lumpectomy, with a 1-2 cm clearance for all cases. For all the patients without clinical evidence of nodal involvement, axillary sampling was done, and lymph node negative status was confirmed on frozen section intra-operatively. For patients with clinically positive nodal disease and those with positive nodes on axillary sampling, complete axillary dissection was performed for level I-III axillary nodes.

\section{Treatment details}

For patients undergoing WBRT, radiotherapy was started either four to six weeks after surgery (for patients not planned for adjuvant chemotherapy), or two to four weeks after completion of adjuvant chemotherapy. Every patient underwent computerized tomography (CT)-based radiotherapy planning. WBRT was delivered with megavoltage photons using 3-dimensional conformal technique (3D-CRT). Hypofractionated dose schedule of $40 \mathrm{~Gy}$ in 15 fractions daily to the entire breast was used, with five fractions a week. This was followed by sequential boost to the tumor bed to a dose of $12.5 \mathrm{~Gy}$ in five daily fractions over one week using enface electrons. Ipsilateral supraclavicular region was also treated to $40 \mathrm{~Gy}$ in 15 fractions for patients who had pathologically node-positive disease or T3/T4 stage of the disease. No patient received axillary radiotherapy or prophylactic internal mammary irradiation.

APBI was performed with multi-catheter interstitial brachytherapy technique using flexible nylon catheters, either intra-operatively or post-operatively after breast-conserving surgery by open cavity technique. Patients' selection criteria, procedure of implant, and dosimetric considerations have been described in detail previously [13]. A dose of $34 \mathrm{~Gy}$ in 10 fractions or $32 \mathrm{~Gy}$ in 8 fractions was prescribed to clinical target volume (CTV), applied twice daily, at least 6 hours apart. APBI began on the third day after intra-operative implant or on the same day as the post-operative implant. If chemotherapy was planned, a gap of two to three weeks was provided between APBI and chemotherapy. For hormone receptor-positive disease, appropriate endocrine therapy was advised as per institutional protocol to all patients.

\section{QOL assessment}

After patient's consent to participate in the study, a single cross-sectional QOL assessment was completed. EORTC QLQ-C30 and BR-23 questionnaires (English and validated translations in Hindi and Marathi) were used for the QOL assessment. QLQ-C30 assesses six function scales (physical, role, emotional, cognitive, social, and global health status) and eight symptom scales (fatigue, nausea/vomiting, pain, dyspnea, insomnia, appetite loss, constipation, diarrhea, and financial difficulties). Breast specific module QLQ-BR23 evaluates four symptom scales (systemic therapy side effects, breast symptoms, arm symptoms, and upset by hair loss) and four functional scales (body image, sexual functioning, sexual enjoyment, and future perspective).

\section{Statistical analysis}

Patients treated with APBI were propensity-score matched to a similar cohort of WBRT using nearest neighborhood algorithm with calliper width of 0.2 for covariates known to effect body image significantly, as described previously [14]. These covariates included menopausal status, size of the surgical cavity $(<100 \mathrm{cc}$ vs. $>100 \mathrm{cc}$ ), size of the tumor in greatest dimension (pathological tumor size $<2 \mathrm{~cm}$ vs. $\geq 2 \mathrm{~cm}$ ), number of lymph nodes dissected, treatment with any chemotherapy (neoadjuvant or adjuvant), and treatment with hormonal therapy. QOL scores for all the items and scales were linearly transformed to $0-100$ points scale, according to the EORTC QOL scoring manual. Mean scores for each domain were analyzed for the entire unmatched cohort as well as compared between the two matched cohorts using Student's two-tailed t-test. A $p$-value of $<0.05$ was considered statistically significant, and a 10-point difference between the mean scores was considered as clinically significant. Mean QOL scores were also compared with EORTC reference values for QOL scores in stage I-II breast cancer, and with mean QOL scores at 3-years follow-up from the GEC-ESTRO randomized trial of APBI vs. WBRT [10]. All statistical analyses were performed using SPSS version 21.0 (IBM Corp.).

\section{Results}

A total of 320 consecutive eligible patients on a follow-up were enrolled into the study from January 2017 to June 2018. Table 1 shows baseline characteristics for these patients.

The patients had received adjuvant RT from January 2015 to November 2016. QOL scores from a single assessment during the time period of 18-36 months after radiotherapy were reported in the present study. The quality-of-life data was collected during the patients' follow-up visits. Of the total 320 patients, 80 were treated with APBI and 240 with WBRT. In the APBI group, $82 \%$ patients received brachytherapy dose of $34 \mathrm{~Gy}$ in 10 fractions, while $18 \%$ received 32 Gy in 8 fractions. In the WBRT cohort, almost all of the patients ( $n=228,95 \%)$ were treated with conventional or 3D-CRT technique us- 
Table 1. Patients' characteristics

\begin{tabular}{|c|c|c|c|c|}
\hline \multirow[t]{2}{*}{ Variable } & \multirow{2}{*}{$\begin{array}{c}\text { Overall } \\
(N=320)\end{array}$} & \multicolumn{2}{|c|}{ Propensity-matched cohort $(N=163)$} & \multirow[b]{2}{*}{$p$-value } \\
\hline & & WBRT $(n=99)$ & $\mathrm{APBI}(n=64)$ & \\
\hline Age (years), mean & $51 \pm 10$ & $55 \pm 7.2$ & $60 \pm 7$ & 0.24 \\
\hline Menopausal status & & & & 0.84 \\
\hline Pre-/peri-menopausal & $112(35 \%)$ & $3(3 \%)$ & $1(1.6 \%)$ & \\
\hline Post-menopausal & $208(65 \%)$ & $96(97 \%)$ & $63(98.4 \%)$ & \\
\hline Diabetes mellitus & $45(14 \%)$ & $18(18 \%)$ & $13(20.3 \%)$ & 0.73 \\
\hline Pathological T stage & & & & 0.33 \\
\hline$\underline{\mathrm{T} 1}$ & $106(33.1 \%)$ & $34(35 \%)$ & $28(43.8 \%)$ & \\
\hline$\underline{\mathrm{T} 2}$ & $210(65.6 \%)$ & $62(62 \%)$ & $36(56.3 \%)$ & \\
\hline $\mathrm{T3}$ & $4(1.2 \%)$ & $3(3 \%)$ & $0(0 \%)$ & \\
\hline Median no. of nodes dissected (range) & $15(1-40)$ & $13(3-36)$ & $10(1-36)$ & 0.23 \\
\hline Cavity volume (cc), median & 110 & 118.1 & 99 & 0.33 \\
\hline Chemotherapy, $n$ (\%) & $309(96.6 \%)$ & $80(80 \%)$ & $44(68.8 \%)$ & 0.28 \\
\hline Hormonal therapy, $n$ (\%) & $195(60.9 \%)$ & $61(61.6 \%)$ & $41(64.1 \%)$ & 0.55 \\
\hline
\end{tabular}

ing bitangential portals. The most common dose prescription for WBRT was 40 Gy in 15 fractions over three weeks $(n=207)$ to the entire breast. Sequential tumor bed boost of 12.5 Gy in 5 fractions over one week was delivered using electrons. Median cavity size was $99 \mathrm{cc}$ and $118.1 \mathrm{cc}$ in the APBI and WBRT cohorts, respectively, as measured in simulation CT images at the time of radiotherapy planning. Any aspiration procedure, if recommended, was performed prior to the simulation CT. Median follow-up was 26 months and 24 months for the APBI and WBRT cohorts, respectively.

Using the propensity-score matching, a total of 64 APBI patients were matched with 99 WBRT patients out of the entire study cohort of 320 cases. Details of propensity-score matching have been reported along with analysis of cosmetic outcomes previously [14]. Mean QOL scores for the matched cohorts of WBRT and APBI are shown in Table 2, and scores for the unmatched cohorts are presented in Appendix 1. Statistically significant difference was observed for QOL scores in the role function, cognitive function, pain, appetite loss, and hair loss sub-scales between the matched WBRT and APBI groups. However, none of the differences in these domains of QLQ-C30 and BR-23 reached clinically significant difference of 10 points, except for the hair loss. There was a small difference of 5-10 points favoring WBRT for the role function (97.0 vs. 91.4, $p=0.015)$, pain $(8.2 \mathrm{vs}$. $16.1, p=0.017)$, and appetite loss (3.7 vs. $10.9, p=0.015$ ) domains. Only a quarter of the patients in the matched cohort $(40$ of 163 ; WBRT $=22, \mathrm{APBI}=18)$ responded to the question of 'upset by hair loss', of which nine reported significant bother ('quite a bit'/'very much'). Mean score for the 'upset by hair loss' sub-scale was significantly higher in the APBI group (51.9 vs. 22.7, $p=0.006)$. However, hair loss is primarily an adverse effect attributable to chemotherapy, and here, out of the quarter of patients reporting any hair loss, $23 \%$ did not receive any chemotherapy. No other factor accounting for hair loss could be identified.

\section{Discussion}

Interstitial brachytherapy is an invasive procedure, and differences in some QOL domains as compared to WBRT can be expected in the first year of follow-up after radiotherapy, as the catheter marks fade and tumor bed heals. Certainly, a few longitudinal studies on APBI assessing change in patients' QOL over time suggested that scores tend to improve over first two years after completion of treatment $[8,15]$. Our study included patients who were 18 to 36 months post-radiotherapy, and by this time, the acute and sub-acute toxicities subsided; therefore, QOL could be expected to stabilize. This might explain the absence of clinically relevant differences in scores of all the QOL domains (i.e., < 10-point difference), despite a few of them reaching statistical significance.

If patient's characteristics were considered for unmatched WBRT and APBI groups, the patients were selected for APBI based on strict recommendations by global professional societies $[2,16]$, which include post-menopausal women with T1-T2 stage, node-negative breast cancer. Large majority of women not fulfilling these criteria are treated with WBRT, along with more extensive surgery and/or more intensive chemotherapy, depending on the tumor stage. Propensity-score matching between APBI and WBRT groups negates the confounding influence of these factors related to patients' selection, tumor stage, and treatment toxicity upon the QOL scores. Moreover, it improves the quality of comparison, even though it reduces the number of observations in order to balance the data. The covariates used for propensity-score matching are the ones shown to significantly influence the body image.

The threshold for clinically meaningful difference used in this study was based on the results of QOL studies from many cancer sites including breast cancer, and estimating a 5-10 points change in QOL score as 'small' or 'trivial' , 10-20 points change as 'moderate', and $>20$ points change as 'large' [17-19]. Alternatively, a threshold of half the standard deviation ( $\mathrm{SD}=0.5$ ) can be used to deter- 
Table 2. Mean quality of life (QOL) scores for matched cohorts

\begin{tabular}{|c|c|c|c|}
\hline \multirow[t]{2}{*}{ QOL domain } & \multicolumn{3}{|c|}{ Propensity-matched cohort $(N=163)$} \\
\hline & WBRT, $n=99$ & APBI, $n=64$ & $p$-value* \\
\hline \multicolumn{4}{|l|}{ QLQ-C30 functional scale } \\
\hline Global health score & $80.0 \pm 16.0$ & $78.6 \pm 15.4$ & 0.586 \\
\hline Physical function & $91.2 \pm 11.8$ & $86.7 \pm 16.1$ & 0.052 \\
\hline Role function & $97.0 \pm 11.0$ & $91.4 \pm 15.7$ & 0.015 \\
\hline Emotional function & $88.6 \pm 19.3$ & $82.9 \pm 20.7$ & 0.080 \\
\hline Cognitive function & $93.1 \pm 13.9$ & $88.3 \pm 14.5$ & 0.037 \\
\hline Social function & $94.4 \pm 16.5$ & $95.1 \pm 11.4$ & 0.781 \\
\hline \multicolumn{4}{|l|}{ QLQ-C30 symptom scale } \\
\hline Fatigue & $19.8 \pm 17.8$ & $23.6 \pm 18.9$ & 0.196 \\
\hline Nausea/vomiting & $4.2 \pm 11.0$ & $4.7 \pm 10.1$ & 0.775 \\
\hline Pain & $8.2 \pm 16.7$ & $16.1 \pm 22.2$ & 0.017 \\
\hline Dyspnea & $5.7 \pm 14.3$ & $7.8 \pm 16.5$ & 0.408 \\
\hline Sleeplessness & $8.4 \pm 23.0$ & $15.1 \pm 23.7$ & 0.078 \\
\hline Appetite loss & $3.7 \pm 13.4$ & $10.9 \pm 20.6$ & 0.015 \\
\hline Financial difficulties & $10.1 \pm 22.1$ & $13.5 \pm 21.2$ & 0.321 \\
\hline \multicolumn{4}{|l|}{ QLQ-BR23 functional scale } \\
\hline Body image & $95.9 \pm 12.1$ & $94.3 \pm 14.1$ & 0.466 \\
\hline Sexual functioning & $6.2 \pm 15.0$ & $4.9 \pm 12.1$ & 0.550 \\
\hline Sexual enjoyment & $39.2 \pm 24.2$ & $33.3 \pm 0.0$ & 0.332 \\
\hline Future perspective & $85.5 \pm 23.4$ & $81.5 \pm 24.5$ & 0.300 \\
\hline \multicolumn{4}{|l|}{ QLQ-BR23 symptom scale } \\
\hline Systemic therapy side effects & $9.3 \pm 11.9$ & $12.2 \pm 13.0$ & 0.151 \\
\hline Breast symptoms & $6.4 \pm 12.5$ & $6.8 \pm 10.4$ & 0.836 \\
\hline Arm symptoms & $11.4 \pm 13.5$ & $13.9 \pm 13.6$ & 0.263 \\
\hline Upset by hair loss & $22.7 \pm 26.0$ & $51.9 \pm 34.7$ & 0.006 \\
\hline
\end{tabular}

${ }^{*} p$ value $<0.05$ and having $\geq 10$-point difference in the score highlighted in bold

mine clinically relevant change [20]. Although the former interpretation of small/moderate/large difference could be simplistic, it is more practical, easier to interpret and compare, and therefore preferred for integrating QOL end points in large clinical trials [10, 21]. The present study demonstrates statistically significant differences in scores for the role and cognitive function, pain, appetite loss, and hair loss, but none, except hair loss, reached the 10-point threshold of clinical significance. The difference in 'upset by hair loss' domain was not attributable to either of the two radiotherapy modalities, occurring most likely due to small number of respondents.

To put our results in context of early breast cancer patients globally, the mean QOL scores observed in the matched pair cohorts of WBRT and APBI in the pres- ent study were compared to the EORTC reference mean QOL scores for stage I-II breast cancer patients, and with the mean QOL scores at 3-years follow-up from the GEC-ESTRO randomized trial of APBI vs. WBRT [10] (Figures 1 and 2). EORTC reference values are available for QLQ-C30 but not for the BR-23 module. Overall, QOL scores in present study were similar or slightly better than the GEC-ESTRO and EORTC reference cohorts, with generally higher function scores and lower symptom scores, except in sexual functioning and sexual enjoyment domains. To note, only $17.6 \%$ patients in our study $(\mathrm{WBRT}=15, \mathrm{APBI}=9)$ reported being sexually active. A comparison of patients' characteristics between the GEC-ESTRO cohort and our institutional APBI group was previously reported [13], and revealed that the patholog- 


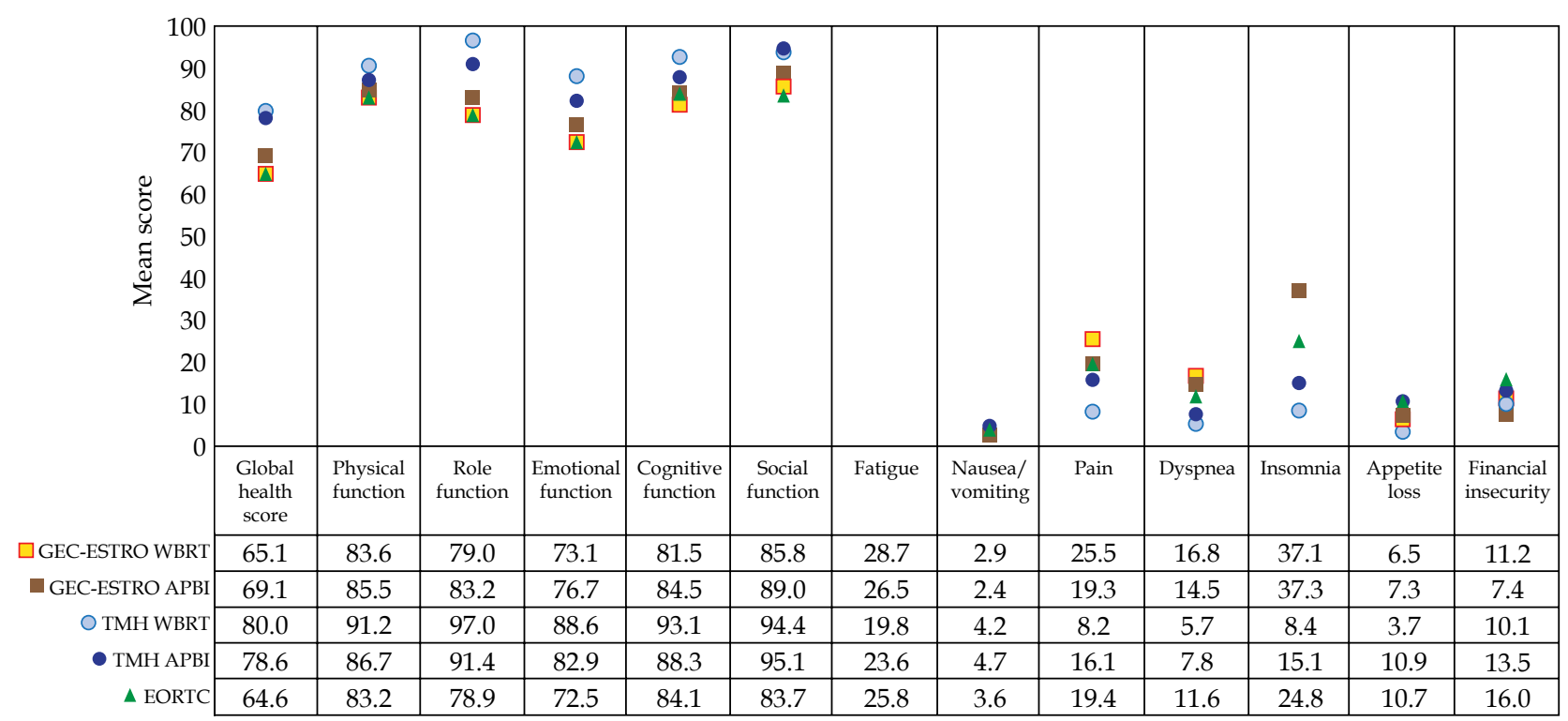

Fig. 1. Comparison of QLQ-C30 mean quality of life (QOL) scores from the propensity score-matched whole breast radiotherapy (WBRT) and accelerated partial breast irradiation (APBI) cohorts of the present study, with the mean QOL scores at 3 years follow-up in the GEC-ESTRO randomized trial of APBI vs. WBRT, and with the EORTC reference mean QLQ-C30 scores for stage I-II breast cancer

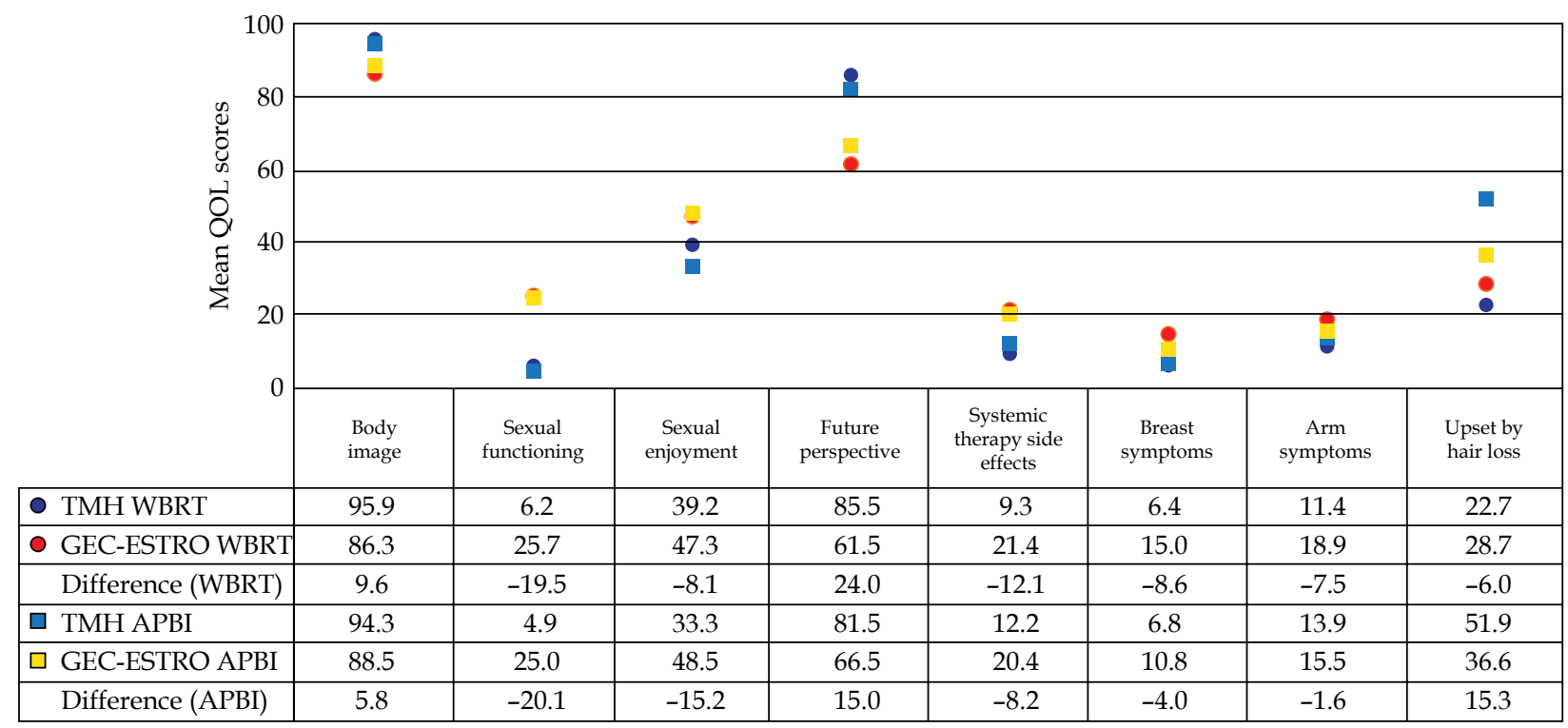

Fig. 2. Comparison of BR-23 mean quality of life (QOL) scores from the propensity score-matched whole breast radiotherapy (WBRT) and accelerated partial breast irradiation (APBI) cohorts of the present study, with the mean QOL scores at 3 years follow-up in the GEC-ESTRO randomized trial of APBI vs. WBRT

ical tumor size tended to be larger and the proportion of estrogen receptor (ER) positive tumors was lower in our patients. These differences also contributed to an increased use of chemotherapy in our patients' cohort. Nonetheless, clinical outcomes were satisfactory, with 7-year disease-free survival of $97.5 \%$ and overall survival of $89 \%$. High level of patient satisfaction with APBI, as observed previously [14], and similar QOL scores in the present study should encourage further uptake of APBI for suitably selected patients with early breast cancer in India. Additionally, it was impossible to embark randomized evidence in Indian setting, especially using brachytherapy because of the lack of availability of the expertise for breast brachytherapy across the country.

Clinical efficacy of APBI has been proven in comparison to WBRT, with high rates of patients' satisfaction and cosmesis [6, 7, 13, 22]. Also, patient-reported QOL has been observed to be similar or better than WBRT in randomized trials as well as prospective studies $[9,10,15,23]$. In addition to multi-catheter interstitial brachytherapy, QOL has also been assessed in patients treated with other APBI techniques. In a study comparing QOL between intensity modulated radiation therapy (IMRT)-based APBI to WBRT, QOL scores up to two years post-radiotherapy 
were better with APBI [23]. Another study showed better QOL scores with APBI using single-lumen and multi-lumen brachytherapy as compared to WBRT [8]. The results from our study are in concordance with the global experience, and confirm good QOL outcomes in Indian patients treated with APBI for localized early breast cancer.

There are some drawbacks to be acknowledged in this study. A single cross-sectional QOL assessment in 18-36 months follow-up period to assess long-term patients' QOL outcomes is simple and practical, but less desirable than longitudinal assessment at multiple time points. The longitudinal assessment of late toxicity and QOL has been initiated as a part of separate prospective study on recently treated patients, and will be published as soon as the data matures. Patients' selection for APBI was based on strict recommendations of global professional societies, which include stage T1-T2 post-menopausal women with node-negative breast cancer $[2,24]$. Patients not meeting these criteria were treated with WBRT, along with more extensive surgery and/or more intensive chemotherapy, depending on the tumor stage. Propensity-score matching between APBI and WBRT groups minimizes the confounding effect of various tumor and treatment factors upon the QOL scores, and improves the quality of comparison. However, the influence of selection bias and unknown confounding variables cannot be completely eliminated. Unlike in Western countries, where screen-detected breast cancer is common and suitable for brachytherapy, early breast cancer patients treated with APBI have larger tumors and higher grade. Regardless of the above, excellent outcomes have been reported for APBI from our institute [25]. Therefore, indirect comparison has been performed with due acknowledgement of various known/unknown biases.

The relevance of small but statistically significant difference in QOL sub-scales between APBI and WBRT can be debated depending on the definition of clinically meaningful difference. Whether such small differences can influence treatment decisions, when considering overall treatment time, resources, and costs associated with APBI, this is something for every radiation oncologist to consider in their own clinical practice.

\section{Conclusions}

Patients with breast cancer undergoing breast-conserving surgery and adjuvant radiotherapy report high QOL scores. The modality of adjuvant radiation (APBI or WBRT) did not have a clinically significant difference in the QOL of patients. Hence, this study supports the use of APBI and higher adoption of this modality in the breast cancer radiotherapy practices in India.

\section{Disclosure}

The authors report no conflict of interest.

\section{References}

1. Correa C, Harris EE, Leonardi MC et al. Accelerated partial breast irradiation: executive summary for the update of an ASTRO evidence-based consensus statement. Pract Radiat Oncol 2017; 7: 73-79.
2. Hepel JT, Arthur D, Shaitelman S et al. American Brachytherapy Society consensus report for accelerated partial breast irradiation using interstitial multicatheter brachytherapy. Brachytherapy 2017; 16: 919-928.

3. Strnad V, Major T, Polgar C et al. ESTRO-ACROP guideline: Interstitial multi-catheter breast brachytherapy as accelerated partial breast irradiation alone or as boost - GEC-ESTRO Breast Cancer Working Group practical recommendations. Radiother Oncol 2018; 128: 411-420.

4. Wadasadawala T, Sarin R, Budrukkar A et al. Accelerated partial-breast irradiation vs conventional whole-breast radiotherapy in early breast cancer: a case-control study of disease control, cosmesis, and complications. J Cancer Res Ther 2009; 5: 93-101.

5. Shah C, Martinez A, Kolar M, Vicini F. Modern approaches for breast brachytherapy. Semin Radiat Oncol 2020; 30: 61-67.

6. Polgár C, Ott OJ, Hildebrandt G et al. Late side-effects and cosmetic results of accelerated partial breast irradiation with interstitial brachytherapy versus whole-breast irradiation after breast-conserving surgery for low-risk invasive and in-situ carcinoma of the female breast: 5-year results of a randomised, controlled, phase 3 trial. Lancet Oncol 2017; 18: 259-268.

7. Coles CE, Griffin CL, Kirby AM et al. Partial-breast radiotherapy after breast conservation surgery for patients with early breast cancer (UK IMPORT LOW trial): 5-year results from a multicentre, randomised, controlled, phase 3, noninferiority trial. Lancet 2017; 390: 1048-1060.

8. Bitter SM, Heffron-Cartwright $\mathrm{P}$, Wennerstrom $\mathrm{C}$ et al. WBRT vs. APBI: an interim report of patient satisfaction and outcomes. J Contemp Brachytherapy 2016; 8: 17-22.

9. Bhattacharya IS, Haviland JS, Kirby AM et al. Patient-reported outcomes over 5 years after whole- or partial-breast radiotherapy: longitudinal analysis of the IMPORT LOW (CRUK/06/003) phase III randomized controlled trial. J Clin Oncol 2019; 37: 305-317.

10. Schäfer R, Strnad V, Polgár C et al. Quality-of-life results for accelerated partial breast irradiation with interstitial brachytherapy versus whole-breast irradiation in early breast cancer after breast-conserving surgery (GEC-ESTRO): 5 -year results of a randomised, phase 3 trial. Lancet Oncol 2018; 19: 834-844.

11. Taylor ME, Perez CA, Halverson KJ et al. Factors influencing cosmetic results after conservation therapy for breast cancer. Int J Radiat Oncol Biol Phys 1995; 31: 753-764.

12. Munshi A, Kakkar S, Bhutani R et al. Factors influencing cosmetic outcome in breast conservation. Clin Oncol ( $R$ Coll Radiol) 2009; 21: 285-293.

13. Budrukkar A, Gurram L, Upreti RR et al. Clinical outcomes of prospectively treated 140 women with early stage breast cancer using accelerated partial breast irradiation with 3 dimensional computerized tomography based brachytherapy. Radiother Oncol 2015; 115: 349-354.

14. Wadasadawala T, Sinha S, Parmar V et al. Comparison of subjective, objective and patient-reported cosmetic outcomes between accelerated partial breast irradiation and whole breast radiotherapy: a prospective propensity score-matched pair analysis. Breast Cancer 2020; 27: 206-212.

15. Garsa AA, Ferraro DJ, DeWees TA et al. A prospective longitudinal clinical trial evaluating quality of life after breast-conserving surgery and high-dose-rate interstitial brachytherapy for early-stage breast cancer. Int J Radiat Oncol Biol Phys 2013; 87: 1043-1050.

16. Polgár C, Limbergen EV, Pötter R et al. Patient selection for accelerated partial-breast irradiation (APBI) after breast-conserving surgery: Recommendations of the Groupe Européen de Curiethérapie-European Society for Therapeutic Radiology and Oncology (GEC-ESTRO) breast cancer working 
group based on clinical evidence (2009). Radiother Oncol 2010; 94: 264-273.

17. Osoba D, Rodrigues G, Myles J et al. Interpreting the significance of changes in health-related quality-of-life scores. J Clin Oncol 1998; 16: 139-144.

18. Snyder CF, Blackford AL, Sussman J et al. Identifying changes in scores on the EORTC-QLQ-C30 representing a change in patients' supportive care needs. Qual Life Res 2015; 24: 1207-1216.

19. Cocks K, King MT, Velikova G et al. Evidence-based guidelines for interpreting change scores for the European Organisation for the Research and Treatment of Cancer Quality of Life Questionnaire Core 30. Eur J Cancer 2012; 48: 1713-1721.

20. Norman GR, Sloan JA, Wyrwich KW. Interpretation of changes in health-related quality of life: the remarkable universality of half a standard deviation. Med Care 2003; 41: 582-592.

21. FAQ. EORTC - Quality of Life. Available at https://qol.eortc.org/faq/ (accessed December 15, 2019).

22. Strnad V, Ott OJ, Hildebrandt G et al. 5-year results of accelerated partial breast irradiation using sole interstitial multicatheter brachytherapy versus whole-breast irradiation with boost after breast-conserving surgery for low-risk invasive and in-situ carcinoma of the female breast: a randomised, phase 3, non-inferiority trial. Lancet 2016; 387: 229-238.

23. Meattini I, Saieva C, Miccinesi G et al. Accelerated partial breast irradiation using intensity modulated radiotherapy versus whole breast irradiation: health-related quality of life final analysis from the Florence phase 3 trial. Eur J Cancer 2017; 76: 17-26.

24. Polgár C, Limbergen E Van, Pötter R et al. Patient selection for accelerated partial-breast irradiation (APBI) after breast-conserving surgery: Recommendations of the Groupe Européen de Curiethérapie-European Society for Therapeutic Radiology and Oncology (GEC-ESTRO) breast cancer working group based on clinical evidence (2009). Radiother Oncol 2010; 94: 264-273

25. Budrukkar A, Telkhade T, Wadasadawala T et al. A comparison of long-term clinical outcomes of accelerated partial breast irradiation using interstitial brachytherapy as per GEC-ESTRO, ASTRO, updated ASTRO, and ABS guidelines. Brachytherapy 2020; 19: 337-347. 
Appendix 1. Mean quality of life (QOL) scores for unmatched cohorts

\begin{tabular}{|c|c|c|c|}
\hline \multirow[t]{2}{*}{ QOL domain } & \multicolumn{3}{|c|}{$\begin{array}{l}\text { Unmatched cohort } \\
\qquad(N=320)\end{array}$} \\
\hline & WBRT, $n=240$ & APBI, $n=80$ & $p$-value \\
\hline \multicolumn{4}{|l|}{ QLQ-C30 functional scale } \\
\hline Global health score & $81.2 \pm 14.7$ & $76.9 \pm 17.6$ & 0.056 \\
\hline Physical function & $91.5 \pm 12.0$ & $85.9 \pm 17.7$ & 0.009 \\
\hline Role function & $96.1 \pm 12.1$ & $92.5 \pm 14.7$ & 0.050 \\
\hline Emotional function & $88.9 \pm 17.5$ & $83.3 \pm 21.8$ & 0.039 \\
\hline Cognitive function & $92.5 \pm 13.5$ & $88.5 \pm 15.1$ & 0.036 \\
\hline Social function & $94.3 \pm 16.1$ & $95.6 \pm 10.5$ & 0.427 \\
\hline \multicolumn{4}{|l|}{ QLQ-C30 symptom scale } \\
\hline Fatigue & $18.1 \pm 17.3$ & $23.2 \pm 19.8$ & 0.042 \\
\hline Nausea/vomiting & $3.6 \pm 9.4$ & $3.9 \pm 9.2$ & 0.773 \\
\hline Pain & $8.3 \pm 15.9$ & $15.6 \pm 21.3$ & 0.005 \\
\hline Dyspnea & $5.7 \pm 13.6$ & $8.3 \pm 16.3$ & 0.196 \\
\hline Sleeplessness & $8.0 \pm 19.5$ & $13.3 \pm 22.9$ & 0.067 \\
\hline Appetite loss & $4.7 \pm 13.8$ & $10.8 \pm 21.7$ & 0.020 \\
\hline Financial difficulties & $13.9 \pm 25.5$ & $12.1 \pm 20.0$ & 0.650 \\
\hline \multicolumn{4}{|l|}{ QLQ-BR23 functional scale } \\
\hline Body image & $95.6 \pm 11.2$ & $94.6 \pm 13.1$ & 0.570 \\
\hline Sexual functioning & $10.0 \pm 18.9$ & $4.4 \pm 11.5$ & 0.002 \\
\hline Sexual enjoyment & $44.4 \pm 26.2$ & $30.6 \pm 9.6$ & 0.003 \\
\hline Future perspective & $81.8 \pm 26.0$ & $82.3 \pm 24.9$ & 0.885 \\
\hline \multicolumn{4}{|l|}{ QLQ-BR23 symptom scale } \\
\hline Systemic therapy side effects & $9.1 \pm 11.1$ & $12.1 \pm 14.2$ & 0.093 \\
\hline Breast symptoms & $5.6 \pm 10.3$ & $7.8 \pm 11.6$ & 0.124 \\
\hline Arm symptoms & $11.3 \pm 11.9$ & $15.7 \pm 16.5$ & 0.031 \\
\hline Upset by hair loss & $35.6 \pm 50.6$ & $51.5 \pm 3.7$ & 0.108 \\
\hline
\end{tabular}

